\title{
L'habitant et ses véhicules aux dix-sept et dix-huitième siècles
}

\section{Robert-Lionel Séguin}

Volume 11, numéro 2, septembre 1957

URI : https://id.erudit.org/iderudit/301834ar

DOI : https://doi.org/10.7202/301834ar

Aller au sommaire du numéro

Éditeur(s)

Institut d'histoire de l'Amérique française

ISSN

0035-2357 (imprimé)

1492-1383 (numérique)

Découvrir la revue

Citer cet article

Séguin, R.-L. (1957). L'habitant et ses véhicules aux dix-sept et dix-huitième siècles. Revue d'histoire de l'Amérique française, 11(2), 242-269.

https://doi.org/10.7202/301834ar d'utilisation que vous pouvez consulter en ligne.

https://apropos.erudit.org/fr/usagers/politique-dutilisation/ 


\section{L'HABITANT ET SES VÉHICULES aux dix-sept et dix-huitième siècles}

\section{LE CANOT}

De nombreux cours d'eau sillonnent la Nouvelle-France. Fleuves et rivières y sont comme les nervures d'une feuille immense. Rares sont les contrées aussi avantageusement dotées de ces avenues qui permettent de s'aventurer jusque dans les coins les plus inviolés. Le Saint-Laurent et ses affluents constituent notre unique système routier, on ne s'étonnera donc pas de voir le Canadien se servir du canot. Aussi, son adresse et son endurance d'avironneur ne sont-elles nulle part contestées. Le chevalier de Beauchesne, étant à Québec, nous raconte qu'il se fait conduire "aisement en Canots jusqu'à Mont-Réal...» ${ }^{1}$ De son côté, le jésuite Lafiteau, qui connaît pourtant bien l'autochtone pour avoir vécu longtemps avec lui, ne manque pas de lui préférer, comme avironneur, «les François Canadiens... si habiles à parer les Roches... » 2 Même que le missionnaire en aurait vu «qui aimoient mieux saulter, le Sault-Saint-Louis ... que de faire le voyage à Montréal à pied. ${ }^{3}$ Cette dextérité ne nous étonne pas, puisque d'après Charlevoix, le Canadien manie l'aviron « dès la Bavette ${ }^{4}$ Enfin, le naturaliste Kalm, en septembre 1749, note que les habitants se fabriquent des canots d'écorce inspirés de la mode indigène et qu'ils « les conduisent à l'indienne ... ${ }^{5}$

Durant la rude saison, le Canadien se déplace en raquettes, de préférence sur les rivières gelées. On y rencontre moins

1 Les Avantures (sic) de monsieur Robert Chevalier, dit de Beauchesne, capitaine de flibustier dans la nouvelle France, (2 vol., à Paris, M.DCC.XXXII), II : 37.

2 P. Lafiteau, Mœurs des Sauvages Ameriquains compare'es aux mœurs des premiers temps, (4 vol., à Paris, MDCCXXIV), III : 199.

3 Ibid.

4 Pierre F.-X. de Charlevoix, Histoire et description générale de la Nouvelle-France avec le journal historique d'Un Voyage fait par ordre du Roi dans l'Amérique septentrionale, (3 vol., Paris, 1744), III : 193.

5 Pierre Kalm, Voyage en Amérique, (2 vol., Montréal, 1880), II : 193. [242 ] 
d'obstacles et l'orientation y est plus facile. Puis, les colons font inlassablement reculer les frontières de la forêt. Chacun piétine son sentier qui s'aboute à celui du voisin. C'est la genèse de notre premier système routier.

L'été, l'habitant voyage d'abord à dos de cheval, mais ce mode de locomotion n'enchante pas les hauts fonctionnaires et les responsables de la colonie. Ils craignent que les Canadiens deviennent paresseux et perdent ainsi leur précieuse qualité de marcheurs. Pontchartrain s'en inquiète l'un des premiers. Aussi, dès le 10 juin 1710, recommande-t-il au gouverneur «de tenir la main à la réduction des chevaux $\gg^{6}$ Le 13 juin précédent, l'intendant Raudot avait donné des instructions encore plus radicales aux habitants de Montréal. Désormais, on «ne pourra avoir plus de deux chevaux ou cavales et un poulin, et ce, à commencer après les semences de l'année mil sept cent dix, leur donnant le dit temps pour pouvoir se défaire des chevaux qu'ils ont au-delà de ce nombre, et après lequel ils seront tenus de tuer ceux qu'ils auraient au-delà. » ${ }^{7}$

Plus tard, le 12 novembre 1712, Vaudreuil et Begon renchérissent sur ces directives. Écoutons-les dire au ministre de la Marine:

la diminution du nombre des chevaux pourra obliger les habitans de la Colonie a aller plus souvent en raquettes, (nous) les y porteront autant que faire ce pourra, estants persuadez comme vous Monseigneur, qu'il est nécessaire de les entretenir dans cet usage qui leur donnera toujours de la supériorité sur les anglois. ${ }^{8}$

Et plus loin, les administrateurs font de nouveau chorus:

Il faut absolument diminuer le nombre des chevaux et remettre les habitans a aller en raquette. Il est de l'interest de ceux qui sont en teste de la colonie que les habitans soient forts et robustes ...9

6 Archives coloniales du Canada, mémoires 1706-1709, fol. 170.

7 Arrêts et règlements du Conseil Supérieur de Québec et Ordonnances et Jugements des Intendants du Canada, (3 vol., Québec, 1854-1856), 2: 273.

${ }_{8}$ Rapport de l'Archiviste de la province de Québec pour 1947-1948, (Québec, 1948), 176, Correspondance entre M. de Vaudreuil et la Cour, 12 9 bre 1712 .

9 Ibid. 
Les mêmes reconnaissent qu'il y a encore trop de chevaux en Canada, dans une lettre adressée au Conseil de la Marine, le 26 février 1717. Le voiturage d'hiver reste une sérieuse objection à la diminution du nombre de ces bêtes, mais diront les autorités, pourquoi ne pas se servir de boufs comme aux premières heures de la colonie ? Ou encore, ne pourrait-on pas simplement chausser les raquettes ? ${ }^{10}$ Malgré de telles exhortations, les temps évoluent et le Canadien ne peut s'en tenir à des moyens de transport aussi primitifs. Nous arrivons aux premiers véhicules.

La voiture canadienne est de fabrication domestique. On se sert du bois des abattis ou, le plus souvent, de celui tiré à dessein de la réserve boisée. Pour cette besogne, l'outillage du terrien est rudimentaire: une scie, un marteau, une hache à équarrir, un rabot, une tarière et une plaine. Pour le reste, le campagnard s'en remet à sa débrouillardise. Et il n'en manque pas, car d'après Charlevoix, on en voyait «tous les jours qui réussissent dans tous les métiers sans en avoir fait l'apprentissage ». ${ }^{11}$ De son côté, Hocquart informe le Ministre que «les habitants font eux-mêmes la plupart des outils et des ustencils de labour, bâtissent leurs maisons, leurs granges... $\gg^{12}$ Beaucoup plus tard, au début du dix-neuvième siècle, un voyageur note à son tour que les Canadiens fabriquent toutes les choses nécessaires à leur usage "ainsi que leurs charrues et leurs canots ». ${ }^{13}$ La même remarque s'applique aux véhicules. Cependant, la fabrication des roues sera confiée au charron de la seigneurie ou à un habitant du rang, reconnu pour son habileté en la matière. Ce qui explique le grand soin que porte le terrien d'alors à ces parties essentielles des voitures.

Dans le choix et la construction de ses véhicules, le campagnard doit considérer la topographie du pays, le genre de culture, le climat et les besognes saisonnières. Nous le verrons s'inspirer,

10 Ibid.

11 Pierre F.-X. de Charlevoix, Histoire et description générale de la Nouvelle-France..., III : lettre VIII.

12 Archives canadiennes, rapport pour l'année 1886, Hocquart au Ministre, Vol. 67, fol. 76.

${ }_{13}$ D. Bainville, Beautés de l'histoire du Canada, etc., (Paris, 1821), 482. 
par obligation, des modes indienne et française. Ces voitures seront d'abord destinées au travail; celles de promenade viendront par la suite. On en conviendra, l'habitant doit d'abord songer aux besognes des champs et de la ferme avant les balades de plaisance. D'autant plus que les routes de l'époque ne lui permettent pas de s'éloigner de sa demeure.

\section{VÉHICULES DE TRAVAIL: LA TRAÎNE}

La Nouvelle-France est un pays froid, où l'on doit chauffer les maisons durant les longs mois de l'hiver. Sous la cognée, le bois se fait de plus en plus rare autour des habitations. Il faut aller le quérir souvent à une bonne distance. Pour le véhiculer, l'habitant fabrique d'abord la traîne. Inspirée de la mode indienne, elle est faite d'orme, quelques fois d'érable, généralement de frêne. Cet arbre présente au moins deux avantages: la flexibilité et la forme d'un coude que prend généralement l'extrémité inférieure de son tronc. Comme la traîne doit transporter des charges lourdes, sur des chemins accidentés, on verra à la construire solide. On choisira d'abord une «botte ${ }^{14}$ de dix pouces à un pied de diamètre, par environ deux ou trois verges de longueur. Bien entendu, il faudra qu'un bout de cette bille, celui qui se trouve à la souche, soit légèrement recourbé. On en coupera un à l'avenant. La pièce sera équarrie, puis on arrondira la recourbure à la hache. Cette opération terminée, le morceau est fendu en deux, généralement à la scie. Nous aurons alors deux patins exactement identiques. On les relie ensuite au moyen de trois au quatre sommiers, placés à égale distance, le tout fortement chevillé. Ce véhicule assez primitif est l'ancêtre de la «traîne à batons » de nos grands pères. On s'en est pareillement inspiré pour fabriquer la «berline » et le « berlot».

Dès 1664, Pierre Boucher nous informe que la traîne est employée au transport du bois. Elle est tirée par des bœufs, car les solipèdes sont rares dans la colonie. ${ }^{15}$ Cette carence n'est

14 Dans la langue campagnarde, la «botte» signifie la première bille prise sur la souche.

15 Pierre Boucher, Histoire Véritable et Naturelle des Mcurs et productions du pays de la Nouvelle France Vulgairement dite Canada (Paris, $1664), 141$. 
que passagère. En 1667, la Mère Marie de l'Incarnation écrira: «Sa Majesté a encore envoyé des chevaux, \& on nous a donné, pour notre part, deux belles juments et un cheval, tant pour la charrue que pour le charroi. ${ }^{16}$ Chose à souligner, la traîne de l'époque n'a pas de lisse ferrée, ce qui ne l'empêche pas d'être maniable, puisqu'elle " glisse sur la neige, et un bœuf seul en mene autant que deux bœufs feroient en Esté dans une charette. » 17

Veut-on savoir le prix généralement payé pour pareil véhicule ? Le 13 avril 1691, "Deux Traisnes Garnis avec un traineau $\gg,{ }^{18}$ trouvés sur la ferme de la veuve St-Ange, à la Pointeaux-Trembles, sont évalués à dix-huit livres.

Nous avons vu que la traîne est destinée au transport lourd. Aussi, sera-t-elle faite de grosses pièces qui la rendent pesante, même sans charge. C'est ainsi qu'à Sainte-Anne-du-Bout-de-l'Ile, le $1^{\text {er }}$ mars 1704, on inhume le corps de Jean Charlebois, décédé trois jours plus tôt, «ayant été écrasé sous une traine ». ${ }^{19}$

Cette voiture subira certaines transformations. Plus tard, le patin sera garni d'une bande de fer, puisqu'en 1760 , il y a "Une Traîne avec lisse », chez Ignace Adam, à Beaumont..0 Il s'en trouve une autre, en novembre 1796, chez les Roque, à Saint-Charles-sur-Richelieu. ${ }^{21}$ Elle vaut six livres et quatre sols, même si nous sommes déjà tout à la fin du siècle.

Par ailleurs, son principal accessoire reste la chaîne qui tient lieu de traits. On utilise une plus petite pour tenir le faix en place. ${ }^{22}$ En novembre 1675, il se trouve des «chesne de trais-

16 Lettres de Marie de l'Incarnation, (Clermont- Ferrand, 1857), 342.

17 Pierre Boucher, Histoire Véritable et Naturelle ..., 141.

18 « Estima on des bestiaux \& au' choses Entre La veuve $s^{\text {t }}$ Ange \& pinsonneau Lafleur son fermier. »13e Avril 1691. Greffe d'Anthoine Adhémar, minute no 1861. En dépôt aux Archives judiciaires de Montréal.

19 Agé de seize ans et demi, le défunt est fils de Jean et de Marthe Perrier, habitants de la paroisse. Ce serait même le premier accident mortel survenu dans le secteur de Vaudreuil. (Cf. Registres de Sainte-Anne-deBellevue, 1704). de Québec.

20 Greffe d'Antoine-Joseph Saillant. En dépôt aux Archives judiciaires

21 Inventaire des Biens de feu Charles Roque et Josette Roi son Epouse. 3 novembre 1796. Greffe de J.-M. Mondelet, minute no 462. En dépôt aux Archives judiciaires de Montréal.

22 Les cultivateurs font la même chose de nos jours. La charge est retenue, à l'avant, par une chaîne, ensuite tendue au moyen d'un petit tronc d'ostryer, placé en tourniquet. 
nes ${ }^{23}$ sur la ferme de Pierre Lorrain, à Montréal. Les «Six Chesnes de Traisnes de fer »qu'on inventorie chez le sieur Jacques Le Ber, en décembre 1693 , sont prisées à trois livres pièce. ${ }^{23 a}$ Le 9 juillet 1695, la chaîne de traîne de Jean Bourdon, de la Prairie de la Magdeleine, est estimée à quatre livres. ${ }^{24}$ Par contre, celle que possèdent les Reid, de Lavaltrie, en janvier 1744, ne vaut que dix sols..$^{25}$ D'autre part, "deux Chaines de traine », ${ }^{26}$ découvertes à la ferme des Labrosse, à la Pointe-Claire, en mars 1779 , sont évaluées à cinq livres.

Dès le début du dix-neuvième siècle, l'habitant se sert d'un autre véhicule pour ses travaux d'hiver. Il s'agit d'une grande voiture, formée de deux trains. En somme, ce sont deux petites traînes, placées l'une devant l'autre et jointes, entre elles, par un court timon. Cet accouplement permet de transporter un plus grand nombre de billes de forte dimension. ${ }^{27}$ Un véhicule semblable est encore couramment utilisé par nos cultivateurs pour charroyer le bois. A tout événement, dès 1814, Lambert nous en fait cette description:

Sleighs are used in the winter, as carts are in the summer, for the transportation of goods. They are formed of a couple of low runners, with boards 'fastened across. The goods are kept from falling off by half-a-dozen sticks, which are fixed at the

23 Bail à ferme par Mons ${ }^{r}$ D'ailleboust a Pierre Lorrain et Sa femme. 1675: 25' Novembre. Greffe Benigne Basset, minute no 1230 . En dépôt aux Archives judiciaires de Montréal.

23a Inventaire des Biens Meubles et Immeubles de la Communauté d'Entre Le sieur Jacques Le Ber Et Dame Jeanne Le Moyne, Sa femme. 1693 et 1694. Greffe Benigne Basset. En dépôt aux Archives judiciaires de Montréal.

24 Inventaire de bourbon. 9 Juillet 1695. Greffe de Claude Maugue, minute no 2917. En dépôt aux Archives judiciaires de Montréal.

25 Inventaire des Biens Des Mineurs Reille deposé Ce jourdhuy 23e janvier 1744. Documents sous seing privé, no 1018. En dépôt aux Archives judiciaires de Montréal.

26 Inventaire des Biens qui Etoient Communs Entre feu pierre La Brosse, Et françoise Lauzon sa Veuve. Le $15^{\mathrm{e}}$ Mars 1779. Greffe de Joseph Soupras, minute no 2261. En dépôt aux Archives judiciaires de Montréal.

27 M. Ovide Voghel, autrefois de Saint-Marc-sur-Richelieu, nous informe que son père, Isidore, de Saint-Hilaire, s'était fabriqué une voiture semblable, en 1878. C'était la première du genre dans la région. Il s'en servait pour le transport du foin, de Saint-Hilaire à Saint-Jean-Baptiste de Rouville. 
sides and corners of the sleigh, and fastened together at top by a rope or twisted twigs. ${ }^{28}$

\section{LA CHARRETTE}

L'habitant a surtout besoin de véhicules pour les tâches printanières, estivales et automnales. A cet usage, nous lui connaissons la charrette et le tombereau. Comme la traîne, ils sont fabriqués sur la ferme, à l'exception des roues qui sortent de la boutique du charron. Par exception, quelques habitants plus doués se chargeront de cette besogne, mais dans ce dernier cas, on laisse quand même à l'artisan le soin de les cercler.

La charrette sera faite d'orme, le plus souvent de frêne, très rarement de chêne. Ce dernier bois n'est pas impropre à pareille fabrication, mais par édit spécial, le roi s'en réserve le droit de coupe pour la construction de ses vaisseaux. ${ }^{29}$

Rappelons que le constructeur de la charrette canadienne s'inspire de la tradition française. Le véhicule est cependant mieux proportionné et de formes plus élégantes que celui dont se sert le paysan européen ou le colonial des possessions espagnoles du Nouveau-Monde. En plusieurs endroits, ceux-ci en sont encore à la roue pleine, tandis que notre habitant n'emploie que celle à jantes, avec rais et moyeu.

Le véhicule canadien n'aura qu'une paire de roues, pour au moins quatre raisons. D'abord par définition, car l'appellation charrette veut dire voiture à deux roues, à ridelles et à limons. Il faut cependant souligner que dans le parler campagnard, le terme est également synonyme de chariot. Mais il y a autre chose en faveur de la première explication. Dans les divers inventaires dressés par les tabellions du temps, il ne sera toujours question que de «deux roues avec ses frettes ». ${ }^{30}$ L'expression est même consacrée dans le vocabulaire notarial. Ensuite

28 John Lambert, Travels through Canada, and the United States of North America, in the years 1806, 1807, \& 1808, (2 vol., London, 1814), 1: 170.

${ }^{29} \mathrm{~A}$ ce sujet, on verra notamment le jugement rendu contre le sieur de Vincelotte, le 4 juillet 1715. (Cf. Arrêts et Règlements du Conseil Supérieur de Québec, etc., 111: 170).

30 Le frette est un lien ou cercle de fer dont on entoure l'extrémité du moyeu des roues pour empêcher d'éclater ou de se fendre. 
le scribe prend soin de spécifier: «avec son essieu de fer ». $\mathbf{A}$ ceci, ajoutons qu'il aurait été difficile de manœuvrer une voiture à double trains sur les sentiers sinueux et dans les champs à peine essouchés de l'époque.

Les voitures à quatre roues ne dateraient vraisemblablement que du début du dix-neuvième siècle. En mai 1806, il se trouve «Un Chariot a quatre roues ferrées » sous le hangar de madame de Longueuil, à l'île Sainte-Hélène. ${ }^{31}$ Il est évalué à dix livres.

L'habitant se sert surtout de la charrette pour la fenaison, les récoltes, le charroi des blés aux établissements banaux et même pour y mener sa famille à l'église. Cette voiture reste « l'ancêtre » de la «charrette à poches » de nos bisaïeuls. Soulignons qu'elle a deux ridelles amovibles à ses extrémités latérales. Le 19 juin 1673, Basset découvre « deux Iroudelles » parmi les biens laissés par Jeanne Mance, dans le grenier de l'hôpital de Montréal. ${ }^{32}$ La première mention d'une voiture du genre, dans les annales montréalaises, remonterait au 8 mars 1664, alors qu'il se trouve « une charrette de foin de quatre bœufs ... ${ }^{33}$ sur le lopin de la veuve Lambert Closse. Il s'agit sans doute d'un véhicule à deux roues, et les bœufs seraient attelés au même timon, par groupes de deux, placés l'un devant l'autre.

Un fâcheux accident nous signale encore l'usage courant de cette voiture, à Montréal, dans le dernier quart du dix-huitième siècle. $\mathrm{Au}$ mois d'août 1680 , Jean Tessier se rend au SaultSaint-Louis, dans la charrette de son père, Urbain Tessier dit Lavigne, un des principaux colons de Ville-Marie. Au cours du trajet, Jean Tessier écrase accidentellement les deux enfants de Jean LeRoy dit LaPensée. Pierre Demay dit LaRose, originaire de Limoges, et René Cuillerier, de Lachine, se trouvent sur

31 Inventaire des biens de la Communauté de feu David Alx. Grant Ecuyer aved Dame Marie Charles Josephe Lemoyne Baronne De Longueuil, Le 31 May 1806. Greffe de Joseph Gray. Archives judiciaires de Montréal.

32 Inventaire des biens meubles, Titres et Enseignements de deffunte Damoiselle Jeanne Mance vivante administratrice de L'hospital de Montréal. - 19 Juin 1673. Greffe Bénigne Basset. Archives judiciaires de Montréal.

33 Bail a ferme par la veufve de feu $\mathbf{S}^{\mathrm{r}}$ Lambert Closse a Pierre Tessier et Anthoine Courtemanche ensemble un desistmt dud bail par les parties. du 8 Mars 1664. Greffe Bénigne Basset. Aux Archives judiciaires de Montréal. 
les lieux. Le conducteur fut-il imprudent ? C'est du moins l'opinion de LeRoy. Le 24 du même mois, les jeunes accidentés reçoivent la visite du chirurgien Antoine Forestier qui se prononce sur la gravité des blessures. Dès le lendemain, LeRoy exige l'emprisonnement du fils Tessier et demande que le père soit condamné à payer les dommages. Le même jour, Urbain Tessier est assigné à comparaître devant le tribunal. Le 26 , on procède à l'interrogatoire de Jean Tessier. Les 27 et 28, Pierre Demay et René Cuillerier viennent déposer que les enfants «ont été blessés par la faute de l'accusé $\gg^{32 a}$ Si les procédures se précipitent d'abord, elles vont languir par la suite. On n'en arrive à un règlement, par arbitrage, que le 6 août 1681. Un certificat, daté du 14 septembre de la même année, atteste qu'Urbain Tessier a payé tous les frais et qu'il se trouve maintenant quitte envers le sieur LeRoy.

Plus tard, le 3 novembre 1667, «Une charette avec Ses roues, Sans bandages... ${ }^{34}$ sont inventoriées à la demeure de Pierre Gadoye, l'un des premiers colons de Ville-Marie. Le 19 juin 1673 , on procède à la visite d'une grange ayant appartenu à Jeanne Mance. Les estimateurs y découvrent «Une charrette garnie de ses roues ferrées et de son essieu de fer ${ }^{35}$ Il y a également «Une roue de charrette ferée de Ses bandes ». ${ }^{36}$ D'autre part, le 26 novembre 1674, Basset dresse la liste des effets que le sieur Remy vient de fournir à son métayer St-Amour. Nous y trouvons la description suivante:

Une Charrette, garnie, de ses roues, ferrées, Son Essieu de fer Une chaisne de fer et ses autres Esquipages, prisée et estimés ensemble a la somme de deux cent livres. ${ }^{37}$

Ce montant fort élevé ne nous surprend pas, surtout si l'on considère que cette voiture est indispensable au colon. Ajoutons

32a Documents sous seing privé. Archives judiciaires de Montréal.

34 Inventaire de deffunt Pierre Gadoye, 1667 3' Novembre - Greffe Benigne Basset, minute no 411. Archives judiciaires de Montréal.

35 Inventaire des biens meubles, Titres et Enseignements de deffunte Damoiselle Jeanne Mance vivante administratrice de L'hospital de Montréal. 1673. 19' Juin. Greffe de Benigne Basset, Archives judiciaires de Montréal. 36 Ibid.

37 Un memoire des Besthiaux, autres meubles Ustancilles fournis aud $S^{t}$ amour coe. fermier par Monsieur Remy du 26' Novembre 1674. Greffe Benigne Basset, minute 1041. Archives judiciaires de Montréal. 
qu'au cours du même inventaire, on relève "cinq ferthes de fer pour la charette », le tout évalué à cinq livres. ${ }^{38}$ L'usage de la charrette se généralise rapidement dans la campagne. C'est ainsi que le $1^{\text {er }}$ juillet 1675, Frontenac ordonnera à tous les habitants de porter désormais leur blé aux moulins seigneuriaux, sous peine de « confiscation des grains et voitures . . . ${ }^{39}$

Le véhicule servira quelquefois à des fins plus lugubres. Le 27 septembre 1681, au soleil couchant, le métayer Jean Brod s'avise de traverser, en canot, une mare qui se trouve en face de la concession des Filles de la Congrégation. Il est accompagné de Pierre Martin, un adolescent de quinze à seize ans. L'embarcation chavire et le domestique se noie. Pour les besoins de l'enquête, son corps est «apporté dans une charette en la Salle de lad Congregation ce Jourdhuy (28 septembre) environ les huit heures du matin . $^{40}$ Le greffier et le substitut au baillage conviennent d'une mort accidentelle.

Naturellement, pour établir le prix d'une charrette, tout dépend de sa fabrication et de l'état dans lequel on la trouve. Aussi, n'ont-elles pas toutes la même valeur que celle des Remy, en novembre 1674. Lorsque le notaire Adhemar se transporte à la Pointe-aux-Trembles, le 13 avril 1691, «La charette Equipée » qu'il y voit n'est évaluée qu'à trente-neuf livres. ${ }^{41}$ Ce fléchissement dans le prix n'est pas attribuable au seul état du véhicule. Nous sommes à la fin du dix-septième siècle et la colonisation connaît un essor sans précédent. Comme on se tourne de plus en plus vers la terre, les instruments aratoires et la charrette deviennent de moins en moins rares, ce qui rabaisse d'autant leur valeur sur le marché. C'est la loi économique de l'offre et de la demande. Cependant, les «deux Charrettes avec leurs roues à

38 Ibid.

39 Arrets et Règlements du Conseil Supérieur de Québec, et Ordonnances et Jugements des intendants du Canada, (Québec, 1855), 62. Ordonnance du Conseil Supérieur, déclarant Banaux les Moulins à vent et à eau, bâtis par les seigneurs, du lundi 1er. juillet 1675 .

40 Verbal de la mort de Jean Broq domestique de la Congrega ${ }^{\text {on }}$. 29 Juin 1681. Greffe de Claude Maugue. Archives judiciaires de Montréal.

41 Estima $^{\text {on }}$ des bestiaux $\&$ au' choses Entre La veuve $s^{t}$ Ange $\&$ pinssonneau Lafleur son fermier. 13e Avril 1691. Greffe d'Anthoine Adhemar, minute no 1861. Archives judiciaires de Montréal. 
bandage et Essieu de fer », inventoriées chez Jacques Le Ber, en décembre 1693, sont prisées à cent cinquante livres pièce. ${ }^{412}$ Cette voiture devient d'usage si courant qu'en 1706, l'intendant Raudot doit émettre une ordonnance qui défend «aux chartiers et voituriers de se tenir sur leurs charettes vuides en allant ou revenant à peine de prison et de dommages et interets... ${ }^{42}$ Nouvelle preuve que la justice du temps reste des plus sévères.

Quoi qu'il en soit, le 4 juillet 1708, Adhemar évalue à vingt livres «Une Charette avec ses roues Garnies de leurs frettes... ${ }^{43}$ Le véhicule, "fort vieux », se trouve sur la ferme de Joseph Dumay. Beaucoup plus tard, le 23 janvier 1744, Nicolas Laurence et Joseph Sanfaçon, respectivement de Saint-Sulpice et de Lanoraie, agissent comme arbitres lors de l'inventaire des biens de Jean-Baptiste Riel, habitant de Lavaltrie. Ils y trouvent "Une Charette avec Ses roues ... » ${ }^{44}$, le tout prisé à treize livres.

Par ailleurs, la charrette n'est pas sans causer certains ennuis à ses conducteurs, du moins dans les rues abruptes de Québec. La Potherie nous dira, vers le même temps, que « les Charettes \& les Carosses néanmoins ont bien de la peine à monter » les chemins de la Haute-Ville. ${ }^{45}$

Enfin, ces voitures cesseront, du moins pour un temps, de servir aux fins pacifiques de l'agriculture. Lorsque le chevalier de Raymond fait l'inspection des postes, en 1754 , il note que "l'entretien des harnois et Charrette » sont à frais communs entre le roi et les négociants. ${ }^{46}$ Durant la guerre de Sept Ans, on va continuer de les affecter à l'approvisionnement des armées. Le 2 août 1758, on rassemblera tous les habitants des environs du

41a Inventaire Des Biens Meubles et Immeubles de la Communauté d'Entre Le sieur Jacques Le Ber Et Dame Jeanne Le Moyne, Sa feme. 1693 et 1694. Archives judiciaires de Montréal.

42 Ordonnances des Intendants et Arrêts du Conseil Supérieur de Québec, (Québec, 1806), 171.

43 Inventaire de Joseph Dumay. 4 Juin 1708. Greffe d'Anthoine Adhémar. Archives judiciaires de Montréal.

44 Inventaire des Biens Des Mineurs Reille depose Ce jourdhuy $23^{e}$ janvier 1744. Documents sous seing privé, no 1018. Archives judiciaires de Montréal.

45 Bacqueville de La Potherie, Histoire de l'Amérique Septentrionale, etc., (4 vol., A Paris, M.DCC.LIII), 1: 233.

$46 R A P Q$, (1927-1928). Mémoire sur les postes du Canada, adressé à M. de Surlaville en 1754, par le Chevalier de Raymond. 
portage de Chambly, afin "qu'ils y amenassent leurs charettes ${ }^{\mathbf{4 7}}$ pour aider les troupes dans leur marche.

Ces véhicules seront encore employés aux travaux de défense, lors de la guerre de la Conquête. Quelques jours seulement avant la désastreuse rencontre des Plaines d'Abraham, le 4 août 1759, Montcalm écrit à Lévis: «Si vous n'aviez pas besoin d'outils, envoyez-nous, avec deux charrettes, avant-midi, cinquante pelles et cinquante pioches ... ${ }^{48}$ Une quinzaine plus tard, le 21, Bougainville informe Montcalm et Vaudreuil qu'il envoie « des détachemens armes pour rassembler toutes les charettes par tout ou on en pourroit trouver pour nos vivres ... ${ }^{49}$ Démarche bien inutile, car à cette période de l'année, les blés sont déjà « javellés » et tous les véhicules servent aux moissons. D'ailleurs, les officiers militaires font tout en leur pouvoir pour assurer la cueillette des grains. Que peuvent faire les armées les mieux disciplinées et les populations les plus déterminées contre la famine ? C'est pourquoi l'on donne les directives suivantes: " ne faites prendre aucune charette, ni chevaux ni harnois que vous n'en fassiez donner un recu et que vous n'ayez par devers vous l'etat et le nom de l'habitant afin de les pouvoir faire rendre aux habitans dans le tems de la récolte. $\gg 50$

D'autre part, l'habitant ne semble pas trop priser son rôle d'agent pourvoyeur des troupes. Le soir de la funeste bataille du 13 septembre, Lévis désespère devant le manque quasi-total de munitions et de denrées. Il aurait fallu, dit-il, en commander du dépôt de Trois-Rivières, mais « il n'y avoit plus de ressource à attendre des charretier du pays ni des charrettes ... ${ }^{51}$

47 Journal du marquis de Montcalm durant ses campagnes en Canada, de 1756 à 175.9 , p. 428.

48 Lettres du marquis de Montcalm au chevalier de Lévis, (Québec, 1894). 4 août 1759.

49 A. Daughty, The Siege of Quebec and the Battle of the Plaine of Abraham, (6 vol., Québec, 1901), IV: 58. - Correspondance de Bougainville, ce 21 Aoust 1759 .

50 A. Daughty, The Siege of Quebec and the Battle of the Plaine of Abraham, (6 vol., Québec, 1901), IV : 58. Correspondance de Bougainville, ce 21 Aoust 1759.

51 Journal des Campagnes du Chevalier de Lévis en Canada, de 1756 à 1760, (Montréal, 1889), 206. 
Les derniers grondements de la canonnade viennent à peine de se faire entendre que la charrette reprend sa place dans notre décor champêtre et continue, comme avant, de servir aux multiples travaux de la ferme. Il arrive même qu'un habitant puisse en posséder deux ou trois, surtout s'il est riche et s'il est propriétaire d'une grande superficie de terrain. C'est le cas d'Ignace Adam, cultivateur de Beaumont. Lorsque ses biens sont mis à l'enchère, en 1760 , les acheteurs ont le choix entre « 2 charrettes montées $\gg .52$

Plus tard, le 8 juin 1766, Jean Drapeau et son épouse, Thérèse Labrèche, font donation de leur terre de la côte Saint-Elzéar, en l'île Jésus. La cession, consentie en faveur de leur fils Charles, comprend aussi tout l'équipement de la ferme, dont « une grande Charaite $\gg .{ }^{53}$

Ce véhicule devient d'usage si courant qu'on le retrouve partout dans la campagne. Seules les roues ont une certaine valeur et trouvent preneurs à bon prix. Elles ont d'ailleurs toujours été recherchées par l'habitant. Rappelons-nous que dès novembre 1675, des enchérisseurs se disputent les "Roues de charrarettes (sic) » en vente à la ferme des Lorrain. ${ }^{54}$ Un siècle plus tard, le terrien accordera toujours le même intérêt aux roues, encore faut-il qu'elles soient en bonne condition. Le 15 mars 1779 , le notaire Soupras, de la Pointe-Claire, inventorie les biens de Pierre Labrosse, habitant du lieu. "Une très Vieille Roue de Charette », trouvée dans la grange, n'est évaluée qu'à deux livres. ${ }^{55}$ Enfin, le 3 novembre 1796, Mondelet fixe à quatorze

52 Vente des biens de Ignace Adam, habitant de Beaumont (1760). Greffe de Saillant. Archives judiciaires de Québec.

53 Donnation par $\mathbf{S}^{r}$ jean drapaux et dame thairaise la breche a charles drapaux Sont fils. le 8 juin 1766. Greffe de C.-F. Coron. Archives judiciaires de Montréal.

54 Bail a ferme par Mons ${ }^{r}$ D'ailleboust a Pierre Lorrain et Sa femme. 1675: 25' Novembre. Greffe Benigne Basset, minute 1230. Archives judiciaires de Montréal.

55 Inventaire des Biens qui Etoient Communs Entre feu pierre LaBrosse, Et françoise Lauzon sa veuve. Le $15^{\mathrm{e}}$ Mars 1779 . Greffe Joseph Soupars, minute 2261. Archives judiciaires de Montréal. 
livres et seize sols, « une Charette et Roues » qui sont alors dans les bâtiments de la famille Roque, à Saint-Charles-sur-Richelieu. ${ }^{56}$

\section{LE TOMBEREAU}

Pour les besoins de la ferme, l'habitant utilise encore le tombereau, également monté sur une paire de roues. Cette voiture, de confection domestique, est faite de frêne, quoique les ridelles et le train soient souvent d'orme ou de pruche. D'inspiration française, le tombereau s'apparente un peu à la charrette, tout en étant plus court et de moindre dimension. En somme, il diffère peu d'avec ceux de notre époque, sauf que les ridelles d'alors sont moins élevées. Ce véhicule sert au transport de la pierre, de la terre et des fumiers. Nous le voyons de bonne heure en Nouvelle-France. Dès juin 1673, un estimateur trouve "Un meschant tombereau, et Une Meschante paire de Roues, sans ferre ${ }^{57}$ parmi les biens laissés par Jeanne Mance, dans les bâtiments de l'hôpital de Montréal. De cette dernière description, doit-on conclure que les roues ne seraient pas cerclées ? A l'instar de certains véhicules du genre, à la mode tant en Europe qu'en Amérique, notre tombereau avait-il des roues pleines ? D'autant plus qu'on ne s'en servait jamais à l'extérieur de la ferme. Nous optons pour le contraire, car à part quelques exceptions, qui sont antérieures au dix-huitième siècle, cette voiture a toujours eu des roues à jantes avec rais et moyeu, tout comme la charrette. Comme d'habitude, la fabrication en est confiée au charron de la seigneurie. Cependant, l'essieu sera taillée à même une "ralle » ${ }^{58}$ d'érable et façonnée par l'habitant lui-même.

Si le tombereau s'avère utile, il n'en est pas moins rare aux premières heures de la colonie. Son prix est donc assez élevé. Le

56 Inventaire des Biens de feu Charles Roque et Josette Roi son Epouse. 3 novembre 1786. Greffe de J.-M. Mondelet, minute 462. Archives judiciaires de Montréal.

57 Inventaire des biens meubles, Titres et Enseignements de deffunte Damoiselle Jeanne Mance vivante administratrice de L'hospital de Montréal. 1673. 19' Juin. Greffe Benigne Basset. Archives judiciaires de Montréal.

58 Dans notre langage campagnard, la « ralle » est une des plus grosses branches d'un arbre. 
13 avril 1691, Adhemar se transporte sur la ferme de la veuve St-Ange, à la Pointe-aux-Trembles, afin d'y estimer toutes les choses qu'elle remet à son fermier Lafleur. « La Charrue avec le tombereau ${ }^{59}$ qui s'y trouvent sont prisés à cinquante-deux livres.

Nouvelle trace de ce véhicule, le 8 novembre de la même année, alors que Louis Lafontaine, habitant de Ville-Marie, promet de travailler pour le compte de Paul Aguenier, menuisier de l'endroit, « avec Ses deux Chevaux et harnois, et equippage de Tombereau avec Ses roues avec une paire de Roues vaccantes, traisne, traisneau ... ${ }^{60}$ L'engagé devra assurer ce service pendant un an, moyennant la somme de deux cent cinquante livres. Plusieurs années passent, puis au début de juin 1766, nous voyons encore un «tombros » en bon état, ${ }^{61}$ cette fois sur la terre des Drapeau, à la côte Saint-Elzéar en l'île Jésus. L'on va continuer d'utiliser cette voiture après la Conquête. Son prix deviendra cependant moins élevé. Le cas suivant nous en convaincra. Louis Séguin, cultivateur de l'Anse de Vaudreuil, meurt à l'automne de 1771. Le 7 octobre de la même année, le tabellion Thomas Vuatier dresse la liste des biens à partager entre ses enfants et sa veuve, née Marie-Anne Raizenne. Sous l'une des remises se trouve «Un Vieux tombereau avec Ces vielle roux frette $\gg,{ }^{62}$ le tout évalué à douze livres.

\section{VÉHICULES DE PROMENADE}

La période de l'essouchement terminée, le campagnard emblave les terres nouvellement nettoyées de leurs chicots calcinés. Le sol est riche. Les générations qui montent connaîtront plus

59 Estima ${ }^{\text {on }}$ des bestiaux \& au' choses Entre La veuve ${ }^{t}$ Ange $\&$ pinssonneau Lafleur son fermier. $13^{\mathrm{e}}$ Avril 1691. Greffe d'Anthoine Adhemar, minute 1861. Archives judiciaires de Montréal.

${ }_{60}$ Marché entre Lafontaine chartier et aguenier. 89 bre 1691 . Greffe de Claude Maugue, minute 2488. Archives judiciaires de Montréal.

61 Donnation par $S^{r}$ jean drapaux et dame thairaise la breche a charles drapaux Sont fils. le 8 juin 1766. Greffe C.-F. Coron. Archives judiciaires de Montréal.

62 Inventaire des Biens de feu Louis Seguin de Vaudreuil en son vivant époux de Marie anne Raizenne. Le $7^{\mathrm{e}} 8^{\mathrm{bre}} 1771$. Greffe de Thomas Vuatier, minute 1142. Archives judiciaires de Montréal. 
d'aisance et vivront dans de meilleures conditions économiques. C'est alors qu'on songera aux voitures de promenade, jusqu'ici considérées comme un luxe.

L'habitant voit défiler les attelages fringants des officiers militaires et civils, alors en tournée dans les paroisses. Il prend goût à cette aisance pourtant relative. Elle blessa même souvent sa vanité. Rien d'étonnant qu'il veuille rivaliser de richesse avec tous ceux-ci, puisque selon l'expression consacrée, le voici devenu «à l'aise ». Bien davantage, il arrive que certains d'entre eux soient les créanciers de fonctionnaires coloniaux les plus huppés, lesquels ne touchent que des appointements insignifiants. A ceci, ajoutons que le Canadien reste un amateur du cheval et un fervent des promenades. Bougainville le confirme, dans son mémoire de 1757: "Tous les habitants, dit-il, c'est ainsi qu'on nomme les paysans en France, ont beaucoup de chevaux et vont toujours en voiture. ${ }^{63}$

Durant les longs mois d'hiver, le cultivateur aura d'abord la carriole, comme voiture de sortie. D'inspiration tout européenne, ce véhicule aux lignes des plus élégantes aurait tout aussi bien sa place sur les avenues de la France métropolitaine que sur les chemins sinueux des seigneuries canadiennes. Mais que sait-on de son aspect? La Potherie nous en fait ainsi la description, tout à la fin du régime français :

Une cariole est une espece de petit carosse coupe par le milieu, \& posee au lieu de roues sur deux pieces de bois, dont les bouts sont recourbez pour glisser plus aisement sur la nege \& sur les glaces. Ces sortes de Voitures sont tres commode, on les embellit de Peinture \& d'Armoiries ... .64

Un peu plus tard, en 1757, Montcalm souligne que le terrien se sert «des voitures appelées carrioles, espèces de traineaux pour aller sur la glace et sur la neige $\gg .65$ Un demi-siècle va s'écouler avant que Lambert nous en parle en ces termes:

63 Journal de M. de Bougainville, 58. Mémoire sur l'Etat de la Nouvelle-France, 1757.

64 Bacqueville de La Potherie, Histoire de l'Amérique Septentrionale, (4 vol., Paris, M.DCC.LIII), 1: 278.

65 Journal de M. de Bougainville, 58. Mémoire sur l'Etat de la Nouvelle-France, 1757 . 
The carioles nearly rewemble the body of a onehorse chaise, palced upon two runners like the irons of a pair of skates. They are painted, varnished, and lined like the better sort of calashes. The driver generally stand up in front, though there is a seat for him similar to that in the calash. Between him and the horse there is a hight pannel which reaches up to his breast, and prevents the splashes from being thrown into the cariole. The body of the vehicle is sometimes placed on high runners of iron, though in general the low wooden runners are preferred, as they are not so liable to be upset as the others. ${ }^{66}$

Ces dernières lignes nous renseignent sur plusieurs particularités de notre carriole. Simplifions : elle n'est, ni plus ni moins, qu'une calèche placée sur patins et à laquelle on a ajouté un devant plus élevé. C'est que la voiture est basse et que les occupants doivent se protéger contre les éclaboussures.

Quand ce véhicule apparaît-il dans la colonie laurentienne? Il semble que ce soit avec le dix-huitième siècle. On le rencontrait d'abord dans les villes. Le 16 août 1709, le sieur Pierre You, de Montréal, troque son cheval avec « un collier, une Cellette, avaloir, Bridon, un travail, une chesne et une Carriolle... $\gg,{ }^{67}$ contre vingt-cinq cordes de bois de chauffage tant " érable, merizier Estre et non Bois Blanc, cedre pruche fresne batard espinette ny sapinage $\gg{ }^{68}$ L'autre partie, Jacques Vaudry, charretier du lieu, devra aussi verser la somme de soixante livres.

De son côté, le jésuite Charlevoix, de passage à TroisRivières le 6 mars 1721, note que les habitants du lieu se servent «d'une Traine, ou comme on parle ici d'une Cariole, qui coule si doucement, qu'un seul Cheval suffit pour la trainer, \& va toujours au galop ${ }^{69}$ Plus tard, l'auteur d'un mémoire sur le

66 John Lambert, Travels through Canada, and the United States of North America, in the years 1806, $1807 \& 1808$, (2 vol., London, 1814), 1: 171 .

67 Vente D'Un Cheval par Me You de La Descouverte A Vaudrey, 16 Avril 1709. Greffe d'Anthoine Adhemar, minute 8200. Archives judiciaires de Montréal.

68 Ibid.

69 Pierre F.-X. de Charlevoix, Histoire et description générale de la Nouvelle-France avec le journal historique d'Un Voyage fait par ordre du Roi dans l'Amérique septentrionale, (3 vol., Paris, 1744), III: 108. 
Canada souligne que les campagnards se promènent dans « des carriolles qui sont des espèces de traineaux accomodes en forme d'un cabriolet ».70 Et le scribe de poursuivre: "c'est un grand plaisir d'aller dans ces voitures et de faire des courses $»^{71}$

Voilà que les militaires veulent aussi se faire véhiculer de la même façon. A la mi-décembre 1753 , un officier et ses compagnons sont invités à une noce qui a lieu à plus de trente milles de Québec. Personne ne veut manquer pareille occasion de s'amuser et le groupe part «en carriole sur la neige $»^{72}$ Plus tard, en février 1757, Montcalm s'inquiète des dépenses considérables encourues par le roi pour le maintien des troupes. Le général ne manque surtout pas de parler des «faux frais en voitures en exprès, en traine, en carrioles dans un pays où tout se paye ... ${ }^{73}$ Il rappelle qu'avec leur solde, "des officiers ont beaucoup de peine à acheter des chevaux et des carrioles ... ${ }^{74}$ Malgré toutes ces remarques, les fonctionnaires n'en continuent pas moins leur vie de pacha. Le 19 mars 1759, Bourlamaque nous décrit, à sa façon, l'entrée de deux d'entre eux à Montréal:

MM. Cadet, Péan arrivés de jeudi, à trois heures après-midi; ils ont fait une entrée d'ambassadeurs, avec douze carrioles, six étoient allées au-devant jusqu'à Repentigny; heureusement le chevalier de Lévis n'en étoit pas. Les relais attendoient sur la glace; $\mathrm{M}^{\mathrm{gr}}$ Cadet qui, pendant qu'on changeoit de relais, donnoit ses audiences aux habitants, de dedans sa carriole, et les relais alloient toujours au galop. ${ }^{75}$

Il appert donc que plusieurs officiers militaires n'ont pas les moyens de se payer une carriole. Ces soucis d'argent n'incommodent cependant pas trop l'habitant, puisqu'on retrouve presque toujours ce véhicule sous sa remise. En 1760 , il y a «Une terres.

${ }^{70} R A P Q,(1933-1934): 212$. Rapport sur la population et la culture des 71 Ibid.

72 Voyage en Canada, par J.B.C., 194.

73 Journal du marquis de Montcalm durant ses campagnes en Canada de 1756 à 1759, 158-159.

74 Ibid. 299.

${ }^{75}$ Lettres de M. de Bourlamaque au Chevalier de Lévis, (Québec, 1891), 
cariolle avec ses menoirs ${ }^{76}$ sur la ferme d'Ignace Adam, de Beaumont. Quelque deux décades plus tard, le 17 mai 1778, Antoine Cédilot dit Montreuil et Marie-Rose Lalonde, tous deux des Cèdres, rédigent leurs conventions de mariage devant le notaire Vuatier. A titre de douaire, le conjoint apporte «Une Cariole aVec Sa ferrure Chaine et traVaille prisé quarante six Livres »..7 Moins d'un an plus tard, Soupras n'estime qu'à douze livres, "Une Vieille Cariolle et ferure dassié sa Chaine Et travail », trouvés chez Pierre Labrosse, à la Pointe-Claire. ${ }^{78}$ Enfin, en novembre 1796, la carriole de Charles Roque, de Saint-Charlessur-Richelieu, vaut vingt-huit livres et dix sols. ${ }^{79}$

Contrairement aux sièges des autres véhicules utilisés par l'habitant, celui de la carriole sera généralement capitonné, quelquefois de fourrure, mais le plus souvent de camelot. ${ }^{80}$ En 1778, « un oreiller de Cariolle de plume CouVert de Camelot rayé », 81 trouvé chez les Montreuil, à Soulanges, est adjugé à huit livres, prix assez élevé si on le compare à celui de la voiture elle-même. L'année suivante, parmi les biens du ménage Labrosse-Lauzon, de la Pointe-Claire, nous trouvons « un oreiller de Cariolle » prisé à quatre livres. ${ }^{82}$ Soulignons que pour se protéger du froid, le voyageur prendra soin de se couvrir d'une couverture. Au début, comme la cueillette des pelleteries s'avère florissante, on se ser-

76 Vente des biens d'Ignace Adam, habitant de Beaumont, 1760. Greffe de Saillant. Archives judiciaires de Montréal.

77 Contrat de Mariage entre $\mathrm{S}^{\mathbf{r}}$ antoine Cedilot $\mathrm{d}^{\mathrm{t}}$ Montreuil et dame marie rose Lalonde. 17 mai 1778. Greffe de Thomas Vuatier, minute 1719A. Archives judiciaires de Montréal.

78 Inventaire des Biens qui Etoient Communs Entre feu pierre LaBrosse, Et françoise Lauzon sa Veuve. Greffe de Joseph Soupras, minute 2261. Archives judiciaires de Montréal.

79 Inventaire des Biens de feu Charles Roque et Josette Roi son Epouse. 3 novembre 1796. Greffe de J.-M. Mondelet, minute 462. Archives judiciaires de Montréal.

80 Il s'agit d'une étoffe qui est d'abord du poil de chameau, puis de chèvre, enfin de la laine et sans grande valeur. Dès 1759 , nos marchands vendent du «camelot de Hollande». (Cf., E.-Z. Massicotte, m.s.r.c., Le Costume Civil Masculin à Montréal au Dix-Septième Siècle, 3e série, 1939, Tome XXXIII : 140.)

81 Contrat de Mariage entre $\mathrm{S}^{\mathrm{r}}$ Antoine Cedilot $\mathrm{d}^{\mathrm{t}}$ Montreuil et dame marie rose Lalonde. 17 mai 1778. Greffe de Thomas Vuatier, minute 1719A. Archives judiciaires de Montréal.

82 Inventaire des Biens qui Etoient Communs Entre feu pierre LaBrosse, Et françoise Lauson sa Veuve. Le $15^{\mathrm{e}}$ Mars 1779 . Greffe de Joseph Soupras, minute 2261. Archives judiciaires de Montréal. 
vira généralement d'une «Robbe de Costor ${ }^{82 a}$ On deviendra moins exigeant par la suite, pour n'employer très souvent qu'une peau d'ours. ${ }^{83}$

Comme la carriole glisse sans bruit sur la neige, il peut en résulter de fâcheux accidents, surtout par une nuit sombre. Pour les éviter, on attache des clochettes aux limons ou à la dossière. Dans certains cas, on n'en accrochera qu'une au collier de la bête. Le bruit des grelots préviendra de tout danger. Cette coutume apparaît vers la fin du dix-huitième siècle. Weld nous en parle ainsi le premier:

The carioles glide over the snow with freat smoothness, and so little noise do they make in slinding along, that it is necessary to have a number of bells attached to the harness, or a person continuallay souding a horn to guard against accidents ...84

Le cri quasi-continuel pour annoncer sa présence sur la route n'a certes pas le charme et la poésie des clochettes. Aussi va-t-on adopter définitivement ces dernières, ce qui contribuera davantage au pittoresque de l'attelage, de même qu'aux promenades à travers la campagne silencieuse.

Rappelons que la carriole peut généralement accommoder deux ou trois personnes. Weld convient que «The Canadian cariole or fledge is calculated to hold two persons and a driver ... ${ }^{85}$ Elle est tirée par un ou deux chevaux. Une grande particularité: dans le dernier cas, les solipèdes sont attelés à la file, car nos chemins d'hiver ont trois ornières et les bêtes trottent au centre. ${ }^{86}$ Cette mode n'est pas sans présenter certains incon-

82a Inventaire des Biens Meubles et Immeubles de la Communauté d'Entre le Sieur Jacques Le Ber et dame Jeanne Le Moyen Sa femme. Le 1er décembre 1693. (Greffe Benigne Basset, minute 2259. Archives Judiciaires de Montréal.

83 Inventaire des Biens qui Etoient Communs entre feu pierre LaBrosse, Et francoise Lauzon sa Veuve, op. cit., Archives judiciaires de Montréal.

${ }^{84}$ Isaac Weld, Travels through the states of North America, and the provinces of Upper and Lower Canada, during the Years 1795, 1796, and 1797. (London, 1799), 226.

85 Isaac Weld, Travels through the states of North America..., (London, 1799), 225 .

${ }^{86} \mathrm{La}$ limonière de côté n'était pas encore de mode. Le milieu de la route était seul battu. 
vénients, voire même certains dangers. Comment maîtriser le cheval placé à l'avant ? De hauts fonctionnaires l'ont compris, puisque le 20 janvier 1742, M. de Monrepos ordonne d'attacher des guides à la bride de la première bête, comme à celle de l'arrière, afin de pouvoir les arrêter en même temps. ${ }^{87}$ Mais pareil harnais n'est pas sans causer quelques ennuis, même aux chevaux. Voici ce que nous en dit le naturaliste Kalm:

Les habitants ont la coutume de couper la queue à leurs chevaux, ce qui est une vraie cruauté ici, puisqu'on les prive ainsi de leur unique moyen de défense contre les moucherons, les taons et les hippobosques. Cette coutume vient peut-être de ce qu'ils attellent leurs chevaux l'un devant l'autre; et pour empêcher celui de devant de blesser les yeux de celui qui est dans les timon en agitant sa queue, ils auront pris le parti d'écourter tous les chevaux. ${ }^{88}$

A ces remarques, ajoutons celles de l'ingénieur Franquet, alors de passage à la Pointe-aux-Trembles, durant l'hiver de 1753:

Les carrioles attelées d'un cheval se conduisent à l'instar de nos calèches en France, mais quand on y en met deux, le second se trouve en avant de l'autre, contenu entre deux traits seulement, de manière qu'il marche suivant sa fantaisie, qu'on ne le ramène au chemin qu'il faut tenir que suivant le mouvement du cheval qui le suit. Cette façon est au retours de celle d'Europe, ou les chevaux de devant dirigent la marche de ceux de derrière ...89

Seule, la bête de l'arrière était placée entre des limons. Celle de l'avant ne traînait le véhicule qu'à l'aide de deux traits. L'épaisseur de la neige, en Canada, nécessitait pareille équipage. Plus loin, le narrateur nous parle de carrioles «trainées par un ou deux chevaux, de la fantaisie d'un chacun .... ${ }^{90}$ Ou encore: «il n'y a que des traineaux pour les équipages et les carrioles à

87 Répertoire des Arrets, Edits, Mandements, Ordonnances et Règlements Conservés dans les archives du Palais de justice de Montréal, (Montréal, 1919), 103.

88 Pierre Kalm, Voyage en Amérique, (2 vol., Montréal, 1880), II : 142. 89 Franquet, Voyage et Mémoires sur le Canada (Québec, 1889), 138. 90 Ibid. 
une personne qui soient attelés d'un seul cheval $\gg^{91}$ De son côté, Bougainville ne s'exprime pas autrement, en 1757: «un seul cheval, dit-il, mène aisement deux personnes dans ces sortes de voitures ».92 Enfin, Weld renchérit ainsi : «it (la carriole) is usually drawn by one horse, if two horses are made use of, they are put one before the other, as the track in the roads will not admit ot their going abreaft $\gg .^{93}$

Soulignons, par ailleurs, que les limons sont généralement à organeaux, c'est-à-dire accrochés à la partie extérieure du patin par des anneaux de fer. Dans les descentes, l'extrémité de la limonière racle le sol et freine ainsi le véhicule dans sa course.

D'autre part, l'endurance du cheval canadien et la légèreté de la carriole permettent de franchir des distances considérables, comme de quinze, vingt-cinq et même trente lieues par jour. C'est ainsi que le 15 mai 1684, La Hontan écrivait de Québec:

On se sert de traineaux, tant à la Ville qu'à la Campagne; pour voitures d'Hyver; les chevaux qui les trainent semblent être de vrayes machines, tant ils sont impénétrables au froid... L'on va d'ici à la Ville de Montréal durant l'Hiver sur le Fleuve par le moyen de traineaux sur lesquels on fait quinze lieues par jour... .94

Weld ne parle pas autrement, un siècle après :

By means of their carioles of fledges, the Canadians transport themselves over the snow, from place to place, in the most agreeable manner, and with a degree of swistness that appears almost incredible; for with the same horse it is possible to go eighty miles in a day, so light is the draft of one of these carriages, and so favourable is the snow to the feet of the horse. ${ }^{95}$

91 Ibid.

92 Journal de M. de Bougainville. Mémoire sur l'Etat de la NouvelleFrance, 1757, 58.

93 Isaac Weld, Travels through the states of North America... (London, 1799), 225.

94 Voyages du baron de LaHontan dans l'Amérique septentrionale...., (2 vol., A Amsterdam, M.DCC.XXXXI), Lettre III, de Québec: 15 May 1684 . 95 Isaac Weld, Travels through the states of North America..., (London, 1799), 225. 
Ces témoignages ne nous surprennent pas, car vers 1738 , Le Beau s'étonne, à son tour, de « la facilité de charroyer du bois à la Ville, sur des traineaux tirés par des Chevaux qui semblent insensibles au froid $\gg .^{96}$ De son côté, le naturaliste Kalm confirme ainsi la vigueur de ces mêmes bêtes: "Tous les chevaux canadiens, dit-il, sont forts, vifs, et bien faits, aussi grands que nos chevaux de cavalerie et d'une race importée de France ${ }^{97}$ On imagine que les accidents soient à craindre avec des bêtes aussi fringantes. Pour les éviter, l'intendant Bégon fait afficher le placard suivant, daté du 29 avril 1716 :

Nous faisons défenses à toutes personnes, tant ceux qui conduiront les Carioles que ceux qui monteront leurs Chevaux, de les faire trotter ou galoper quand ils sortiront de l'Eglise, avant d'en être éloignée de dix arpents, ensuite pourront donner à leurs Chevaux le train qu'ils voudront, lorsqu'il n'y aura personne devant eux, ni charois ni traine, leur ordonnons lorsqu'ils trouveront des gens de pied dans leur Chemin, de s'arrêter et même de se détourner afin de leur donner le tems de se retirer, le tout à peine de vingt livres d'amende $\ldots .^{98}$

Toutes ces précautions ne sont pas exagérées. Nous sommes en présence d'un de nos premiers règlements destinés à assurer une plus grande sécurité routière. D'autre part, l'habitant se promène-t-il autrement qu'en carriole ? A ce sujet, Le Beau écrit que durant la première moitié du dix-huitième siècle, les cultivateurs des rives du Saint-Laurent « ont leur maison si éloignée les unes des autres, qu'ils sont obligés pendant l'Eté, de monter à cheval, \& durant l'Hiver de se servir de traineaux pour aller à leurs Eglises $\gg^{99}$ Dans ce dernier cas, il s'agirait vraisemblablement de la traîne. Bougainville fait servir ce véhicule

96 Avanture (sic) du $S^{r}$ Le Beau, avocat en parlement ou Voyage curieux et nouveau..., (2 vol., A Amsterdam, MDCCXXXVIII), I : 81.

97 Pierre Kalm, Voyage en Amérique, (2 vol., Montréal, 1880), II : 142.

98 Ordonnances des Intendants et Arrets portant reglements du Conseil Supérieur de Québec, (Québec, 1806), 71. - Ordonnance qui défend aux habitants de faire galoper leurs chevaux et leurs Carioles à la sortie de l'Eglise. Michel Begon, \&c. 29 février 1716.

${ }_{99}$ Avanture (sic) $d u S^{r}$ Le Beau, avocat en parlement ou Voyage curieux et nouveau..., (2 vol., A Amsterdam, MDCCXXXVIII), I: 62. 
à d'autres usages. Dans un mémoire de 1757 , il précise que «le transport des marchandises se fait l'été en barques ou canots et l'hiver en traineaux ».100 Cette voiture sera bientôt entourée de panneaux de planches, souvent amovibles, et servira aux sorties de toute la famille. Nos campagnards la désigneront sous l'appellation de "berline ». ${ }^{1}$ En 1814, Lambert nous en parle ainsi :

The Habitans make use of an old-fashioned sort og cariole, called a berlin; it is better adepted for long journeys, as the sides are higher and keep the traveller warmer than the other description of carioles. $^{2}$

Tous ces moyens de locomotion ne suffiront pas, puisqu'on va recourir aux attelages de chiens. Dès 1684, La Hontan nous informe qu'on se transporte, de Montréal à Québec, sur des traîneaux tirés par de gros dogues. Certains n'utilisent que deux bêtes. Aussi, voyagent-ils plus lentement que les autres. ${ }^{3}$

Ce mode de transport semble s'être conservé jusqu'à la fin du régime. Des religieux l'emprunteront pour aller de mission en mission. Le Beau, qui nous visite vers 1740 , nous dira à ce sujet:

Les Recollets se servent aussi d'une autre espèce de traineaux qu'ils font tirer par des gros Dogues, lorsqu'ils vont à leur quête. La glace du Fleuve St. Laurent, a bien souvent dix pieds d'épaisseur, \& si quelquefois le vent en ballaye la neige qui la couvre, Les Habitants vont alors avec une grande facilité, depuis Quebec jusqu'à Montréal: autrement ils se servent de Raquettes à la manière des Sauvages ... ${ }^{4}$

D'autre part, comme la température estivale favorise les déplacements, le Canadien va continuer, pour un certain temps,

100 Journal de M. de Bougainville. Mémoire sur l'Etat de la NouvelleFrance, 1757, 58.

1 En Europe, la berline était une voiture de promenade à quatre roues.

2 John Lambert. Travels through Canada, and the United States of North America, in the years 1806, 1807, \& 1808, (2 vol., London, 1814), I: 171.

${ }^{3}$ Voyages du baron de La Hontan dans l'Amerique septentrionale.... (2 vol., A Amsterdam, M.DCC.XXXI). Lettre III, de Québec, 15. May 1684 . ${ }^{4}$ Avanture (sic) du $S^{r}$ Le Beau, avocat en parlement ou Voyage curieux et nouveau..., (2 vol., A Amsterdam, MDCCXXXVIII), I:81. 
de se transporter en canot ou à dos de cheval. Ce n'est que plus tard qu'il aura des voitures de promenade, pour la belle saison. A cause des ornières et du mauvais état des routes, la calèche va jouir de la faveur populaire. Grâce à leur grande circonférence, les roues du véhicule s'embourbent moins facilement. Vers la moitié du dix-huitième siècle, on retrouve déjà couramment cette voiture dans les villes. Si bien que le 22 mars 1749, Monrepos ordonne de nettoyer les rues de Montréal et défend d'y laisser "nuitamment des calèches, traines, charettes et autres embarras ... ${ }^{5}$ Cette voiture sera vite introduite dans les campagnes où on la rencontre fréquemment à la fin du régime. Le 12 mai 1756, faute de vents contraires, Montcalm débarque à la Petite-Ferme. Un habitant reconduit le général à Québec, dans une «des petites voitures du pays, charrettes ou calèches, qui, aux dires du militaire, sont comme nos cabriolets, conduites par un seul cheval ${ }^{6}{ }^{6}$

Et Montcalm d'en profiter pour vanter les qualités du coursier. "L'espèce des chevaux, poursuit-il, est dans le goût de ceux des Ardennes pour la force, la fatigue et même la tournure. Le chemin de la Petite-Ferme à Québec est beau; on le fait dans la belle saison en six heures, on change à chaque paroisse de voiture, ce qui retarde, à moins qu'on n'en ait fait prévenir. On paye ces voitures à un cheval à raison de vingt sols par lieue. $\gg^{7}$ Dernière précision: "Les lieues sont déterminées sur celles de l'Ile-deFrance $\gg .8$

Une telle chaîne de relais laisse supposer que la calèche soit déjà d'usage courant dans les campagnes. D'ailleurs, Bougainville le confirme l'année suivante: "L'été on (l'habitant) se sert toujours de voitures appelées calèches, et ressemblant aux cambiatieres d'Italie.... ${ }^{9}$

5 Repertoire des Arrets, Edits, Mandements, Ordonnances ..., (Montréal, 1919), 166. (Reg. des éd. et ord., 1743-56, 84).

6 Journal du marquis de Montcalm durant ses campagnes en Canada de $1756 \grave{a} 1759,61$.

7 Journal du marquis de Montcalm ..., 61.

8 Ibid.

9 Journal de M. de Bougainville. Mémoire sur l'Etat de la NouvelleFrance, 1757, 58. 
Restent enfin les harnais. En Nouvelle-France, on emploie Indifféremment ceux à traits ou à fetons. La dernière façon, plus pratique, est préférée à la première jusqu'à la fin du dixhuitième siècle. Précisons: les voitures d'hiver, comme la traîne et la carriole, sont tirées à traits; tandis que celles d'été, comme la charrette, le tombereau et la calèche, sont attelées à fetons. Les véhicules à deux roues, du moins pour la plupart, sont attelés de cette façon, ce qui facilite la manœuvre, pour le conducteur et pour la bête.

Cependant, comme nous nous servons d'abord de voitures d'hiver, il semble que nos premiers harnais soient à traits. Nous en trouvons deux, chez les Le Ber, à Montréal, en $1693 .^{9 \mathrm{a}}$ Le $1^{\text {er }}$ mai 1702, un nommé Alavoyne, de Montréal, loue un cheval à un habitant du lieu, nommé François Coron. A la fin du bail, celui-ci devra remettre l'animal «Avec Les Collier bende \& traitz . $^{\mathbf{1 0}}$

Nous trouvons des attelages à fetons, au même endroit, moins d'une quinzaine d'années plus tard. Le 11 avril 1716, Jacques Denoyon afferme sa terre à Léger Martin. Le marché comprend aussi certains agrès nécessaires à l'exploitation de la ferme. A ce propos, le tabellion Tailhandier ne manque pas de souligner que le locateur a «Livré aud preneur deux Coliers a Chevaux tous neuf sortant de La main de Louvrier Un Es bien garny de fettons Et Couplet de cuir neuf Un bridon tout neuf Une daussieres neuve avec des boucLes de fer une avaloire Et Les Chaine Le tout neuf Une paire de Courroyes...» ${ }^{11}$ Tout cet équipement, pourtant en bon état, n'est évalué qu'à quatre livres. Dans les biens de Jean-Baptiste Riel, habitant de Lavaltrie, inventoriés le 23 janvier 1744, nous relevons «Une vieille avaloir garnie de Ses chaines une vielle dosiere, avec Ses porte

9a Inventaire des Biens Meubles et Immeubles de la Communauté d'Entre le Sieur Jacques Le Ber Et dame Jeanne Le Moyne Sa feme. Le $1^{\text {er }}$ decembre 1693. Greffe Benigne Basset, minute 2259. Archives judiciaires de Montréal.

10 Bail a Louage dun cheval par $\mathrm{M}^{\mathrm{r}}$ Alavoyne a $\mathrm{f}^{\mathrm{s}}$ Caron, premier may 1702. Greffe d'Antoine Adhemar, minute 6080. Archives judiciaires de Montréal.

11 Bail de La ferme à Jacques denoyon donnée à Leger martin Le $11^{\mathrm{e}}$ avril 1716. Greffe Marien Tailhandier, minute 580. Archives judiciaires de Montréal. 
fais un Cossin, Deux vielles brides Leurs mors et guaines, un viel Colier avec Ses chevilles de fer ...»,12 le tout prisé à quatorze livres. Plus tard, en 1760 , nouvelle trace d'un attelage à bacul. "Une paire de traits », ${ }^{13}$ trouvé chez Ignace Adam, à Beaumont, sera évaluée à quatre livres. Il arrive souvent qu'un futur conjoint apporte plusieurs pièces de harnais en douaire. C'est signe d'une certaine aisance et le geste est bien vu des campagnards. Un bel exemple du genre nous est donné le 17 mai 1778, alors qu'on rédige les conventions du mariage qui sera célébré entre Antoine Cédilot dit Montreuil et Marie-Rose Lalonde, tous deux des Cèdres. Le conjoint possède plusieurs choses, entre autres:

Un harnois picquet aVec Ces Chaine Cellette dociere et porte fais prisé Ensemble aVec la Bride picqué à auarante huit Livres.

Un Collier aVec Ces fetons prisé dix Livres

Un Collier a porter prise trois liVres. ${ }^{14}$

L'année suivante, le 15 mars 1779, «Un Vieu harnois, Et deux Colliers ${ }^{15}$ trouvés dans les bâtiments de Pierre Labrosse, à la Pointe-Claire, valent vingt-neuf livres.

A ce propos, rappelons que le collier est de fabrication domestique. On se sert de cuir provenant de peaux d'animaux abattus ou morts sur la ferme, puis traitées à la plus proche boutique de tanneur. Le collier est bourré de filasse. Pour cette opération, on utilise une sorte de machine qui consiste en une large planche, à l'extrémité de laquelle on a fixé une pièce de bois taillée suivant la forme d'un cou de cheval. Un câble, enroulé autour, se durcit au moyen de blocs en coulisse et d'un tourniquet. Ce mécanisme,

12 Inventaire des Biens Des Mineurs Reille déposé Ce jourdhuy 23e janvier 1744. Sous seign privé, document no 1018. Archives judiciaires de Montréal.

13 Vente des biens d'Ignace Adam. Greffe Saillant (Cf. BRH, vol. 62, no $3: 137)$.

${ }_{14}$ Contrat de Mariage entre $\mathrm{S}^{\mathrm{r}}$ antoine Cedilot $\mathrm{d}^{\mathrm{t}}$ Montreuil et dame marie rose Lalonde. 17 mai 1778. Greffe de Thomas Vuatier, minute 1719A. Archives judiciaires de Montréal.

15 In ventaire des Biens qui Etoient Communs Entre feu pierre LaBrosse, Et françoise Lauzon sa Veuve. Le 15e Mars 1779. Greffe Joseph Soupras, minute 2261. Archives judiciaires de Montréal. 
assez ingénieux, facilite de beaucoup le bourrage du collier. Quant aux attelles, elles sont également faites par l'habitant, avec du bois provenant de sa terre. On les ferre ensuite à la forge, avec des vieux morceaux de métal.

Il reste que les voitures et les attelages de l'habitant canadien peuvent rivaliser, tant pour le confort que pour la richesse, avec ceux de tout autre paysan d'Europe. Bien plus, les véhicules que nous venons de décrire, témoignent d'une aisance et d'un confort qu'on ne rencontre pas toujours chez le campagnard de l'époque.

Rigaud, P.Q.

ROBERT-LIONEL SÉGUIN

Articles à paraître dans nos prochaines livraisons :

$1^{\circ}$ Robert Le Blant: Notes sur les découvreurs français de l'Amérique du Nord au XVI siècle. - Inventaire des principales pièces indiquées par les Arrêts du Parlement de Rouen du 22 juin 1633.

$2^{\circ}$ Joseph Cossette, s.j., Jean Talon, champion au Canada du gallicanisme intégral.

$3^{\circ}$ L.-Ph. Cormier (de l'Université de Michigan), La Presse française de l'Illinois.

$4^{\circ}$ Georges-J. Joyaux (de l'Université de Michigan), Xavier Marmier et l'Amérique.

$5^{\circ}$ Antonio Langlais, Messieurs de Saint-Sulpice devant le Conseil Souverain en $166 \%$.

$6^{\circ}$ Fernand Ouellet: Les causes économiques des événements de 1837-1838.

$7^{\circ}$ Germain Lavallée; Monseigneur Antoine Racine et la question universitaire canadienne (1875-1892) .

Maints autres articles et documents inédits. 